\title{
Computation of Period Matrices of Real Algebraic Curves*
}

\author{
Mika Seppälä \\ Department of Mathematics, University of Helsinki, \\ Hallituskatu 15, 00100 Helsinki, Finland \\ mika.seppala@helsinki.fi
}

Dedicated to the memory of Mario Raimondo

\begin{abstract}
In this paper we derive a numerical method which allows us to compute periods of differentials on a real algebraic curve with real points. This leads to an algorithm which can be implemented on a computer and can be used to study the Torelli mapping numerically.
\end{abstract}

\section{Introduction}

The real moduli space, $\bar{M}_{\mathrm{R}}^{g}$, of stable real algebraic curves of a given genus $g$, $g>1$, is a compact Hausdorff space [8]. Let $M_{\mathrm{R}}^{g}$ be the subset of $\bar{M}_{\mathrm{k}}^{g}$ consisting of points corresponding to smooth real algebraic curves of genus $g$. The space $M_{\mathrm{R}}^{g}$ has $\lfloor(3 g+4) / 2\rfloor$ connected components. Each component of $M_{\mathrm{R}}^{g}$ is a real analytic and semialgebraic variety of dimension $3 g-3$ [9].

Let $\mathscr{A}_{\mathrm{R}}^{g}$ be the moduli space of principally polarized real Abelian varieties of dimension $g$. This space is a real analytic and semialgebraic variety having $\lfloor(3 g+2) / 2\rfloor$ connected components [9, Theorem 6.1]. In [12] Silhol has constructed a "natural" compactification $\mathscr{A}_{\mathrm{R}}^{g}$ for $\mathscr{A}_{\mathrm{R}}^{g} . \overline{\mathscr{A}}_{\mathrm{R}}^{g}$ is a connected and compact Hausdorff space.

The components of $M_{\mathrm{R}}^{g}$ correspond to the different topological types of real algebraic curves. Real algebraic curves without real points, especially, form one component of $M_{k}$. This component is a special case in our considerations and has to be excluded in most of the following.

\footnotetext{
* This work was supported by the EC Science Plan Contract No. SC1*.CT91-0716 and the Academy of Finland.
} 
Let $M_{\mathrm{RR}}^{g} \subset M_{\mathrm{R}}^{g}$ and $\bar{M}_{\mathrm{RR}}^{g} \subset \bar{M}_{\mathrm{R}}^{g}$ be the subsets corresponding to the real isomorphism classes of real curves with real points. The Torelli mapping $\tau: M_{\mathrm{k}}^{g} \rightarrow \mathscr{A}_{\mathrm{R}}^{g}$ takes the real isomorphism class of a real curve $C$ onto that of its Jacobian variety $J_{C}$. By the real form of the Torelli theorem [4], [11], the mapping $\tau$ is an injective real analytic mapping. The mapping $\tau$ maps different components of $M_{\mathrm{RR}}^{g}$ into different components of $\mathscr{A}_{\mathrm{R}}^{g}$. The component $M_{\mathrm{R}}^{g} \backslash M_{\mathrm{RR}}^{g}$, which is the set of isomorphism classes of real curves without real points, is a special case. The Torelli mapping maps that component of $M_{\mathrm{R}}^{g}$ onto a component which corresponds to some component of $M_{\mathrm{RR}}^{g}$.

The correspondence, furnished by the Torelli mapping, between the moduli spaces $M_{\mathrm{RR}}^{g}$ and $\mathscr{A}_{\mathrm{R}}^{g}$ is highly transcendental. The aim of this paper is to provide a theoretical basis for a computer program that can be used to study this correspondence. At a later stage we plan to use it, in particular, to compare the compactified moduli spaces $\bar{M}_{\mathrm{RR}}^{g}$ and $\overline{\mathscr{A}}_{\mathrm{R}}^{g}$ and to study the behavior of the Torelli mapping at the infinity of $M_{\mathrm{RR}}^{g}$.

The above setting is the motivation for this paper. It is our intention to develop methods which allow us to compute (numerically by a computer) the image of the real isomorphism class of a real algebraic curve (with real points) under the Torelli mapping. To this end we need:

- A numerical way to define a real algebraic curve (with real points).

- An algorithm to compute a period matrix of an algebraic curve given numerically.

There are, of course, two basically different ways to define a smooth algebraic curve numerically:

- We can give polynomials that define the curve as a subset of a projective space.

- We can express the curve (or, better, the corresponding Riemann surface), via the uniformization, as the unit disk modulo the action of a finitely generated Fuchsian group.

In this note we use the latter approach. Silhol has considered the computation of a period matrix of a real curve defined by polynomials.

It turns out that we can use the following two ways of defining a genus $g, g>1$, smooth real algebraic curve $C$ with real points:

- We can either represent $C$ in the form $\Omega(G) / G$ where:

$-G$ is a Fuchsian group of the second kind freely generated by $g$ hyperbolic Möbius transformations mapping the unit disk onto itself, $-\Omega(G)$ is the domain of discontinuity of $G$,

- or $C$ has an unramified double covering $C^{\mathrm{d}}$ which can be written in the above form $C^{\mathrm{d}}=\Omega(G) / G$. $C$ can then be studied via this double covering $C^{\mathrm{d}}$.

For us, the generators of the group $G$ form the numerical data defining a real algebraic curve with real points. The paper is organized in such a way that we first show how Poincare series can be used to find a basis for the space of Abelian differentials of the Riemann surface $\Omega(G) / G$ for a Fuchsian group $G$ of the second 
kind. This is actually an old observation by Burnside [2]. We follow the exposition of Earle and Marden [3].

Then we show how that can be applied to computing periods of differentials.

In the final sections we consider the error that we make when we replace the infinite Poincare series, appearing in the computation, by their first $n$ elements. ${ }^{1}$

We conclude the paper by considering an implementation of this algorithm in simple cases. These implementations have been developed in collaboration with Apiola and Haataja.

The methods of this paper allow us to compute period matrices of symmetric Riemann surfaces only. In a recent manuscript [10] together with Silhol we present a way to compute period matrices of general compact Riemann surfaces numerically. From the computational point of view, the general algorithm developed in [10] is much more complicated than that of this paper.

A user-friendly interface to this and other similar programs is presently under development at the University of Helsinki in collaboration with Klaus-Dieter Semmler in Lausanne. This interface allows the creation of various kinds of Fuchsian groups in a simple manner from elementary parts corresponding to spheres with three boundary components or torii with one boundary component. The interface allows Dehn twists to be performed to the Fuchsian groups thus created. Other similar operations are also possible. The interface is written in $\mathrm{C}$ and it uses X $11 \mathrm{R} 4$ (or later) and Motif 1.1 (or later).

\section{Real Curves and Symmetric Riemann Surfaces}

Geometrically a smooth real algebraic curve $C$ is a compact Riemann surface $X$ together with an antiholomorphic involution $\sigma: X \rightarrow X$. This involution is induced by the complex conjugation. A Riemann surface $X$ which has an antiholomorphic involution is called symmetric. Any symmetric compact Riemann surface is a smooth real algebraic curve. This correspondence between real algebraic curves and symmetric Riemann surfaces was already observed by Klein [6] and has been investigated in detail in [1].

For our applications it is convenient to classify smooth real algebraic curves or symmetric Riemann surfaces $(X, \sigma)$ into three classes:

- Curves without real points or empty real curves. They are symmetric Riemann surfaces $(X, \sigma)$ for which the involution $\sigma$ does not have fixed points. Such real algebraic curves do not have any real points.

- Orthosymmetric real curves are symmetric Riemann surfaces $(X, \sigma)$ for which $X \backslash X_{\sigma}$ has two components. Here $X_{\sigma}$ denotes the fixed-point set of $\sigma: X \rightarrow X$.

- Diasymmetric real curves are symmetric Riemann surfaces $(X, \sigma)$ such that $X_{\sigma}$ is nonempty and $X \backslash X_{\sigma}$ has only one component.

\footnotetext{
${ }^{1}$ In our first tests we have been able to compute approximately 100,000 first elements of the series in question using an APL2 running on an IBM RS6000 equipped with 16 Mbytes of memory and having about $100 \mathrm{Mbytes}$ of free disk space that were used for temporary files. The computations were done quickly taking only a few seconds.
} 
Sometimes empty real curves are considered to be special cases of orthosymmetric Riemann surfaces. It is, however, more convenient for us to consider them as a separate class.

Let $(X, \sigma)$ be an orthosymmetric real curve and let $Y$ be a component of $X \backslash X_{\sigma}$. It is immediate that $Y$ is a (noncompact) Riemann surface with ideal boundary components which correspond to the fixed-point set $X_{\sigma}$ of $\sigma$. Recall that components of the fixed-point set of an antiholomorphic involution are always simple closed geodesic curves on $X$. Therefore, the Riemann surface $Y$ is the interior of a bordered Riemann surface $\bar{Y}$.

We have assumed that the genus of $X$ is at least 2. Then $Y$ can be expressed as $D / G$ where $G$ is a freely generated group of Möbius transformations acting in the unit disk $D$. Group $G$ is of the second kind, i.e., the domain $\Omega(G)$ of the discontinuity of $G$ is connected and symmetric with respect to the unit circle $\partial D$. The reflection in $\partial D$ is an antiholomorphic involution mapping $\Omega(G)$ onto itself. Call this mapping $\tilde{\sigma}$. Then $\tilde{\sigma}(z)=1 / \bar{z}$.

It follows that $X=\Omega(G) / G$ and the reflection $\tilde{\sigma}: \Omega(G) \rightarrow \Omega(G)$ induces the symmetry $\sigma: X \rightarrow X$. This is the representation that we use to study orthosymmetric real curves.

Assume now that $(X, \sigma)$ is diasymmetric. Consider the quotient $Y=X /\langle\sigma\rangle$. Since $(X, \sigma)$ is diasymmetric, $Y$ is a nonorientable Klein surface. Since $(X, \sigma)$ has real points, i.e., since $\sigma: X \rightarrow X$ has fixed points, $Y$ is a bordered Klein surface. Let $Y^{\circ}$ be the orientable double covering of the nonorientable Klein surface $Y$. The Klein surface $Y^{0}$ is an orientable bordered Klein surface having an antiholomorphic involution $\tau: Y^{\circ} \rightarrow Y^{\circ}$ such that $Y=Y^{\circ} /\langle\tau\rangle$ where $\langle\tau\rangle$ is the group generated by $\tau$.

Let $X^{\mathrm{s}}$ be the Schottky double of $Y^{\circ}$. It is obtained by gluing $Y^{\circ}$ and its mirror image (complex conjugate) together along the boundary curves. The involution $\tau: Y^{\mathbf{o}} \rightarrow Y^{\mathbf{0}}$ induces an antiholomorphic involution $\tau: X^{\mathbf{S}} \rightarrow X^{\mathbf{S}}$ and $X=X^{\mathrm{s}} /\langle\tau\rangle$. This is obvious by the construction. For more details about the Schottky double and other double coverings see, e.g., [1].

It is important that $X^{S}$ is now an orthosymmetric Riemann surface. It can, therefore, be expressed as $X^{\mathbf{S}}=\Omega(G) / G$ for a Fuchsian group $G$ of the second kind. This orthosymmetric Riemann surface has an additional symmetry induced by the symmetry $\tau$ of the orientable double covering $Y^{\mathbf{0}}$. This symmetry acts on $X^{\mathbf{S}}$ and $X=X^{\mathbf{S}} /\langle\tau\rangle$. This construction allows us to apply the methods, that we first develop for orthosymmetric Riemann surfaces, to the diasymmetric case.

\section{Poincaré Series}

In a seminal paper Poincaré [7] introduced, in 1884, a series which can be used to approximate $m$-forms, $m>1$, of a Kleinian group $G$. In that paper Poincaré also observed that these series, which since then have been called Poincare series, do not converge in general for $m=1$. In the case of Fuchsian groups corresponding to compact Riemann surfaces, especially, Poincaré series cannot be used to approximate 1 -forms. 
Seven years later Burnside observed [2] that the Poincare series (of weight 1) converge if the Fuchsian group in question is of the second kind. We use this observation by Burnside to develop an algorithm which allows us to approximate a period matrix of a symmetric compact Riemann surface. This algorithm allows us to study numerically the behavior of the real Torelli map between the moduli space of stable real algebraic curves and the moduli space of principally polarized real Abelian varieties.

Here we review some results and proofs contained in the paper Earle and Marden [3]. This is done for the convenience of the reader: the methods of Earle and Marden play an important role in estimating the errors of our approximations.

Let $D=\{z \in C|| z \mid<1\}$ be the unit disk and let $G$ be a Fuchsian group acting in $D$. Let $Y=D / G$. Assume now that $G$ is of the second kind. This means that the domain of discontinuity of $G, \Omega(G)$, is connected. Then $X=\Omega(G) / G$ is a symmetric Riemann surface, the symmetry $\sigma: X \rightarrow X$ being the mapping induced by the reflection in the unit circle $\partial D$. All orthosymmetric Riemann surfaces can be expressed in this way.

Use the notation $D^{1}(X)$ for the complex vector space of holomorphic Abelian differentials on $X$. Holomorphic differentials $\omega$ on $X$ lift to automorphic holomorphic functions $\omega: D \rightarrow C$ satisfying

$$
\omega(g(z))\left(\frac{\partial g(z)}{\partial z}\right)=\omega(z)
$$

for all $z \in D$ and for all $g \in G$. This is, of course, quite standard.

The limit set, $L(G)$, of a Fuchsian group $G$ of the second kind is a subset of $\partial D$ of measure 0 . The Riemann surface $Y=D / G$ is an open Riemann $Y$ surface which is the interior of a Riemann surface $Y \cup \partial Y$ with boundary components.

These boundary components correspond to half-open intervals in $\partial D \backslash L(G)$. Let $I \subset \partial D$ be a maximal union of these half-open intervals satisfying

$$
g(I) \cap I=\varnothing
$$

for all $g \in G \backslash\{I d\}$. Then

$$
\partial D=L(G) \cup\left(\bigcup_{g \in G} g(I)\right) .
$$

In the following estimates, in particular in Section 5, set $I$ plays an important role. In all occasions set $I$ can be chosen in any way one wants such that (2) and (3) hold. In particular $I$ can be chosen (provided that one knows how) to have maximal measure among all sets $I$ satisfying (2) and (3). Such an $I$ gives us the best error-estimates in Section 5. 
By (3) and by the fact that $L(G)$ is of measure 0 , we obtain

$$
\begin{aligned}
2 \pi=\int_{\partial \boldsymbol{D}}|d z| & =\sum_{\boldsymbol{g} \in \boldsymbol{G}} \int_{\boldsymbol{g}(I)}|d z| \\
& =\int_{I}\left(\sum_{\boldsymbol{g} \in \boldsymbol{G}}\left|\frac{\partial g(z)}{\partial z}\right|\right)|d z| .
\end{aligned}
$$

Set

$$
\rho(z)=\sum_{g \in G}\left|\frac{\partial g(z)}{\partial z}\right|
$$

Equation (4) now implies that $\rho(z)<\infty$ a.e. in $I$.

Lemma 1. The series (5) converges uniformly on every compact subset of $\hat{C}$ which does not intersect the closure of $G(\infty)=\{g(\infty) \mid g \in G\}$.

Proof [3, Proposition 2, p. 204]. Let $G=\left\{g_{1}, g_{2}, g_{3}, \ldots\right\}$ with $g_{1}=$ Id. Each $g_{n}$ is of the form

$$
g_{n}(z)=\frac{a_{n} z+b_{n}}{\bar{b}_{n} z+\bar{a}_{n}}, \quad\left|a_{n}\right|^{2}-\left|b_{n}\right|^{2}=1
$$

Since no element of $G$ has a fixed point in $D, b_{n} \neq 0$ for $n>1$. For $z \in \partial D$,

$$
\left|\frac{\partial g_{n}(z)}{\partial z}\right|=\left|\bar{b}_{n} z+\bar{a}_{n}\right|^{-2} \geq\left(\left|b_{n}\right|+\left|a_{n}\right|\right)^{-2} \geq\left(2\left|a_{n}\right|\right)^{-2} \text {. }
$$

Since $\rho(z)$ is finite for some $z$, we have

$$
\sum_{n}\left|a_{n}\right|^{-2}<\infty
$$

and since $\left|a_{n}\right|^{2}-\left|b_{n}\right|^{2}=1$,

$$
\sum_{n=2}^{\infty}\left|b_{n}\right|^{-2}<\infty
$$

Let $K \subset \hat{C}$ be a compact set disjoint from the closure of $G(\infty)$.

$$
\delta=\text { distance of } K \text { from the closure of } G(\infty) \text {. }
$$

Observe that the closure of $G(\infty)$ is $G(\infty) \cup L(G)$. By our assumptions $\delta>0$. 
For $z \in K$ and $n>1$,

$$
\begin{aligned}
\left|\frac{\partial g_{n}(z)}{\partial z}\right| & =\left|b_{n}\right|^{-2}\left|z+\bar{a}_{n} \bar{b}_{n}^{-1}\right|^{-2} \\
& =\left|b_{n}\right|^{-2}\left|z-g_{n}^{-1}(\infty)\right|^{-2} \\
& \leq \delta^{-2}\left|b_{n}\right|^{-2}
\end{aligned}
$$

from which Lemma 1 follows.

Corollary 1. $\rho(z)$ is a bounded continuous function on $\partial D$ satisfying $\rho(g(z))=\rho(z)$ for all $z \in \partial D$ and for all $g \in G$.

Let $G$ be a Fuchsian group acting in the unit disk $D$ and let $f: D \rightarrow \widehat{C}$ be a meromorphic function. Let $m \geq 1$ be an integer. Consider the Poincaré series

$$
\Theta^{m}(f)(z)=\sum_{g \in G} f(g(z))\left(\frac{\partial g(z)}{\partial z}\right)^{m}
$$

If this series converges, then its limit is a meromorphic function $\omega$ satisfying

$$
\omega(g(z))\left(\frac{\partial g(z)}{\partial z}\right)^{m}=\omega(z)
$$

Use the notation $\Theta(f)(z)=\Theta^{1}(f)(z)$.

It has already been observed by Poincare that, for finitely generated Fuchsian groups of the first kind, the series (9) with $f(z)=z$ converges provided that $m>1$ and diverges if $m=1[7$, p. 308]. Using the Poincare series it is therefore possible to find differentials of order at least 2 on any Riemann surface. However, we are concerned with Abelian differentials.

Lemma 1 implies the convergence of $\Theta^{1}(f)(z)$ in the case of Fuchsian groups of the second kind provided that the function $f$ is not too bad.

Lemma 2. Let $f$ be a rational function with no poles in $L(G)$. Then $\Theta(f)(z)$ is a meromorphic differential of the group $G$.

Proof [3, Proposition 3, p. 205]. Let $K \subset \hat{C} \backslash L(G)$ be a compact set containing the poles of $f$. Since $K \subset \Omega(G), g(K) \cap K \neq \varnothing$ for only a finite number of elements of $G$. Let

$$
M=\sup _{z \in \mathcal{C} \backslash \mathbf{K}}|f(z)| .
$$


It follows that, for all $g \in G$ for all $z \in K,|f(g(z))|<M$ with possibly a finite exception. We conclude, by Lemma 1 , that $\Theta(f)$ is a meromorphic function on $K$.

This is an interesting result which allows us to approximate Abelian differentials of groups of the second kind numerically. The above argument is taken directly from the paper by Earle and Marden but the result itself was already contained in a paper by Burnside [2] that was published in 1891. Since the paper by Burnside appeared the terminology has changed. Burnside refers to Fuchsian groups of the first kind as being of the second kind and vice versa.

Assume now that group $G$ is freely generated by the hyperbolic Möbius transformations $g_{1}, g_{2}, \ldots, g_{p}$ mapping the unit disk onto itself. For $j=1,2, \ldots, p$ let

$$
h_{j}(z)=\frac{\bar{\zeta}_{j}}{1-\bar{\zeta}_{j} z}, \quad \zeta_{j}=g_{j}(0), \quad 1 \leq j \leq p .
$$

Lemma 3. $\left\{\Theta\left(h_{j}\right) \mid 1 \leq j \leq p\right\}$ is a basis for holomorphic differentials on $X=\Omega(G) / G$.

Proof. See Corollary 1, p. 208, of [3].

\section{Estimating the Periods}

Using Lemma 3 we can rather easily estimate periods of differentials. To that end we have to integrate the base differentials along simple closed curves on $\Omega(G) / G$. These curves can, furthermore, be taken to be geodesic curves. Consider such a curve $\alpha$. Choose a lifting of $\alpha$ to the cover $\Omega(G)$. Denote this lifting also by $\alpha$. It is now an arc in $\Omega(G)$, which is disjoint from the closure of the limit set $L(G)$ of $G$.

Lemma 4. The integral of a base differential $\Theta\left(h_{j}\right)$ along $\alpha$ can be computed by the convergent series

$$
\int_{\alpha} \Theta\left(h_{j}\right) d z=\sum_{g \in G} \log \left(\frac{1-\bar{\zeta}_{j} g(\alpha(0))}{1-\bar{\zeta}_{j} g(\alpha(1))}\right)
$$

Proof. By Lemmata 1-3, the Poincaré series $\Theta\left(h_{j}\right)$ converges uniformly on $\alpha$. We 
have therefore,

$$
\begin{aligned}
\int_{\alpha} \Theta\left(h_{j}\right) d z & =\int_{\alpha}\left(\sum_{g \in G} \frac{\bar{\zeta}_{j}}{1-\bar{\zeta}_{j} g(z)} \frac{\partial g(z)}{\partial z}\right) d z \\
& =\sum_{g \in G}\left(\int_{\alpha} \frac{\bar{\zeta}_{j}}{1-\bar{\zeta}_{j} g(z)} \frac{\partial g(z)}{\partial z} d z\right) \\
& =\sum_{g \in G}\left(-\int_{g(x)} \frac{-\bar{\zeta}_{j}}{1-\bar{\zeta}_{j} w} d w\right) \\
& =\sum_{g \in G}\left(\log \frac{1-\bar{\zeta}_{j} g(\alpha(0))}{1-\bar{\zeta}_{j} g(\alpha(1))}\right) . \quad \square
\end{aligned}
$$

In estimating the sum (14) we have the following problems:

(1) Since we can use only finite precision, we can deal with a small part of $G$ at one time only. This part that is available for our computation depends on the choice of the basis of $G$. Long words of elements of $G$ get, as their numerical approximations, identity mappings. They do not contribute anything to the sum (14).

(2) Rounding errors.

We can, however, improve our algorithm using the following construction. The arc $\alpha$, along which we integrate, can often be taken to be an arc on the axis $A_{g_{\alpha}}$ of some Möbius transformation $g_{\alpha} \in G$. We may, furthermore, suppose that the orientation of $A_{g_{\alpha}}$, as determined by the attracting and the repelling fixed points $a_{g_{x}}$ and $r_{g_{\alpha}}$, agrees with that of $\alpha$. For if that is not the case, then replace $g_{\alpha}$ by its inverse. We may also suppose, without restricting the generality, that:

- $g_{\alpha}$ is primitive in $G$, i.e., that the cyclic subgroup $G_{\alpha}=\left\langle g_{\alpha}\right\rangle$ of $G$ generated by $g_{\alpha}$ contains all elements of $G$ which have $A_{g_{\alpha}}$ as their axis.

- $g_{a}(\alpha(0))=\alpha(1)$. This follows from the fact that, in our applications, the projection of $\alpha$ on the Riemann surface $\Omega(G) / G$ is a simple closed curve. We may, therefore, suppose that $g_{\alpha}$ maps the starting point of $\alpha$ onto its endpoint.

Having made all the above assumptions, we now observe that:

(1) $m\left(g(\alpha) \cap g^{\prime}(\alpha)\right)=0$ for all $g, g^{\prime} \in G_{\alpha}, g \neq g^{\prime}$. Here $m$ denotes the one-dimensional Lebesgue measure.

(2) $A_{g_{\alpha}}=\bigcup_{g \in G_{\alpha}} g(\alpha)$.

By the above properties we now have

$$
\sum_{g \in G_{x}}-\int_{g(\alpha)} \frac{-\bar{\zeta}_{j}}{1-\bar{\zeta}_{j} \omega} d \omega=-\int_{A_{\theta_{\alpha}}} \frac{-\bar{\zeta}_{j}}{1-\bar{\zeta}_{j} \omega} d \omega=\log \frac{1-\bar{\zeta}_{j} r_{g_{\alpha}}}{1-\bar{\zeta}_{j} a_{g_{\alpha}}}
$$


Therefore, by (15), we may compute an infinite part of the series (14) by a single expression only. This improves the accuracy of our computations and reduces rounding errors considerably.

Let now $g \in G \backslash G_{\alpha}$. The arc $\beta=g(\alpha)$ lies on the axis of the Möbius-transformation $g_{\beta}=g \circ g_{\alpha} \circ g^{-1}$.

The subgroup $G_{\beta}=\left\langle g_{\beta}\right\rangle$ of $G$ contains all transformations of $G$ whose axis agrees with that of $g_{\beta}$. Also in this case we have:

(1) $A_{g_{\beta}}=\bigcup_{h \in G_{\beta}} h(\beta)$.

(2) $m\left(h(\beta) \cap h^{\prime}(\beta)\right)=0$ for all $h, h^{\prime} \in G_{\beta}, h \neq h^{\prime}$.

Hence

$$
\begin{aligned}
\sum_{h \in G_{\beta}}-\int_{h(g(\alpha))} \frac{-\bar{\zeta}_{j}}{1-\bar{\zeta}_{j} \omega} d \omega & =-\int_{A_{g \beta}} \frac{-\bar{\zeta}_{j}}{1-\bar{\zeta}_{j} \omega} d \omega \\
& =\log \frac{1-\bar{\zeta}_{j} r_{g_{\beta}}}{1-\bar{\zeta}_{j} a_{g_{\beta}}}
\end{aligned}
$$

By (15) and (16) we conclude that periods of differentials can be computed by the formula

$$
\int_{\alpha} \Theta\left(h_{j}\right) d z=\sum_{h \in G / G_{\alpha}} \log \frac{1-\bar{\zeta}_{j} h\left(r_{g_{\alpha}}\right)}{1-\bar{\zeta}_{j} h\left(a_{g_{\alpha}}\right)}
$$

which is, in numerical computation, much more accurate and faster than formula (14).

\section{Estimating the Errors}

Let $f$ be a rational function with no poles in the closed unit disk. Let $G$ be a Fuchsian group of the second kind acting in the unit disk and generated by the hyperbolic Möbius transformations $g_{1}, \ldots, g_{p}$. Let $G=\left\{I d=g_{1}, g_{2}, g_{3}, \ldots\right\}$ where the enumeration is chosen in such a way that for $m>n$ the word-length of $g_{m}$ (in terms of the generators and their inverses) is at least as large as that of $g_{n}$.

By Lemma 2,

$$
\omega(z)=\sum_{n=1}^{\infty} f\left(g_{n}(z)\right) \frac{\partial g_{n}(z)}{\partial z}
$$

converges and represents a holomorphic differential on $D / G$. In this section we derive an estimate for the error that we make when we replace the infinite series (18) by its first $m$ terms. 
In estimating the sum of the series (18) it would be desirable to be able to order the elements of $G$ is such a way that if $m>n$, then the corresponding multipliers of Möbius transformations satisfy $k\left(g_{n}\right) \geq k\left(g_{m}\right)$. Elements $g$ with small multipliers contribute more to the sum (18) than those with large multipliers. Unfortunately it is not clear how this can be achieved in the general case. ${ }^{2}$

For a positive integer $m$, let

$$
\begin{array}{ll}
\rho^{m}:=\sum_{n=1}^{m}\left|\frac{\partial g_{n}(z)}{\partial z}\right|, & \\
A^{m}:=\sum_{n=1}^{m}\left|a_{n}\right|^{-2}, \quad A:=\sum_{n=1}^{\infty}\left|a_{n}\right|^{-2}, \\
B^{m}:=\sum_{n=1}^{m}\left|b_{n}\right|^{-2}, \quad B:=\sum_{n=1}^{\infty}\left|b_{n}\right|^{-2} .
\end{array}
$$

Lemma 5. For any $m, a z \in \bar{I}$ exists such that

$$
0 \leq \rho(z)-\rho^{m}(z) \leq \frac{1}{m(I)} m\left(\partial D / \bigcup_{n=1}^{m} g_{n}(I)\right)
$$

where $m$ is the one-dimensional Lebesgue measure on the unit circle.

Proof.

$$
\begin{aligned}
m\left(\partial D / \bigcup_{n=1}^{m} g_{n}(I)\right) & =\sum_{n>m} \int_{g(I)}|d z| \\
& =\sum_{n>m} \int_{I}\left|\frac{\partial g}{\partial z}\right| \\
& =\int_{I}\left(\rho(z)-\rho^{m}(z)\right) \\
& \geq m(I) \min \left(\rho(z)-\rho^{m}(z)\right) .
\end{aligned}
$$

Since $\rho$ is a bounded and continuous function, $\rho-\rho^{m}$ attains its minimum at some point of the closure of the set $I$. Estimate (20) then implies that the statement of the lemma holds for this point $z$.

The next lemma, on the other hand, follows directly from (6).

\footnotetext{
${ }^{2}$ Nielsen's algorithm can be used to find, for $G$, a set of generators whose multipliers are small. It is not known whether this algorithm leads to the best generating set.
} 
Lemma 6. We have

$$
0<A-A^{m} \leq \frac{1}{4 m(I)} m\left(\partial D / \bigcup_{n=1}^{m} g_{n}(I)\right)
$$

Let

$$
\begin{aligned}
& m_{G}(z):=\sup _{g \in G, g \neq 1}|z-g(\infty)|, \\
& M_{G}:=\sup _{z \in D} m_{G}(z) .
\end{aligned}
$$

Since $G$ acts properly discontinuously also in the exterior of the unit disk, $M_{G}<\infty$. In our applications it is usually possible to estimate the constant $M_{G}$ in terms of the generators of $G$.

Since

$$
\begin{aligned}
\left|\frac{\partial g_{n}(z)}{\partial z}\right| & =\left|b_{n}\right|^{-2}\left|z+\bar{a}_{n} \bar{b}_{n}^{-1}\right|^{-2} \\
& =\left|b_{n}\right|^{-2}\left|z-g_{n}^{-1}(\infty)\right|^{-2} \\
& \geq\left|b_{n}\right|^{-2} M_{G}^{-2}
\end{aligned}
$$

(see (8)) we have

$$
\left|b_{n}\right|^{-2} \leq M_{G}^{2}\left|\frac{\partial g_{n}(z)}{\partial z}\right|
$$

Hence we have:

Lemma 7. Let $z \in \bar{I}$ be the point of Lemma 5:

$$
0<B-B^{m} \leq M_{G}^{2}\left(\rho(z)-\rho^{m}(z)\right) \leq \frac{M_{G}^{2}}{m(I)} m\left(\partial D / \bigcup_{n=1}^{m} g_{n}(I)\right)
$$

Proof. The lemma follows from estimate (21) and Lemma 5.

The next estimate concerns the differential $\omega$. Let

$$
\omega^{m}(z)=\sum_{n=1}^{m} f\left(g_{n}(z)\right) \frac{\partial g_{n}(z)}{\partial z}
$$


Lemma 8. The error of the estimate $\omega^{m}$ for $\omega$ at a point $z \in D$ satisfies

$$
\left|\omega(z)-\omega^{m}(z)\right| \leq \frac{M_{f} M_{G}^{2}}{m(I)(1-|z|)^{2}} m\left(\partial D / \bigcup_{n=1}^{m} g_{n}(I)\right)
$$

where $M_{f}:=\sup _{z \in D}|f(z)|$.

Proof. We have

$$
\begin{aligned}
\left|\omega(z)-\omega^{m}(z)\right| & =\left|\sum_{n>m} f\left(g_{n}(z)\right) \frac{\partial g(z)}{\partial z}\right| \\
& \leq M_{f} \sum_{n>m}\left|\frac{\partial g_{n}(z)}{\partial z}\right| \\
& \leq M_{f} \sum_{n>m} \delta^{-2}\left|b_{n}\right|^{-2} \\
& \leq M_{f} \frac{1}{(1-|z|)^{2}}\left(B-B^{m}\right) \\
& \leq \frac{M_{f} M_{G}^{2}}{m(I)(1-|z|)^{2}} m\left(\partial D / \bigcup_{n=1}^{m} g_{n}(I)\right) .
\end{aligned}
$$

Estimate (24) follows from (8). Estimate (25) follows from estimate (8) and from the fact that the limit set of $G$ lies on the boundary of the unit disk $D$.

The above error estimates involve constants related to the group $G$. They are $M_{G}$ and $m(I)$. In practical examples $M_{G}$ can often be estimated directly by knowing the generators of the group. We do not, however, have any algorithmic way of doing this for an arbitrary group $G$ given in terms of its generators. The reason is that the generators need not be transformations having small multipliers. Their multipliers can be arbitrarily large and there is no clear way of finding those elements of the group whose multipliers are the smallest.

Estimating the constant $m(I)$ also involves a detailed study of the action of group $G$. It suffices to find any set $I$ satisfying the defining conditions (3) and (2). We would, of course, like to choose $I$ in such a way that $I$ is as large as possible. Often that can be done in the way we have described in Fig. 1. In the figure, $I$ is simply the intersection of the fundamental domain in question and the unit circle. It is clear that in the case of this figure the set $I$ is the largest possible.

\section{An Example}

Lemmata 2-4 form an algorithm for computing an estimate for a period matrix of a real algebraic curve with real points. Lemma 8 furnishes a way to estimate 


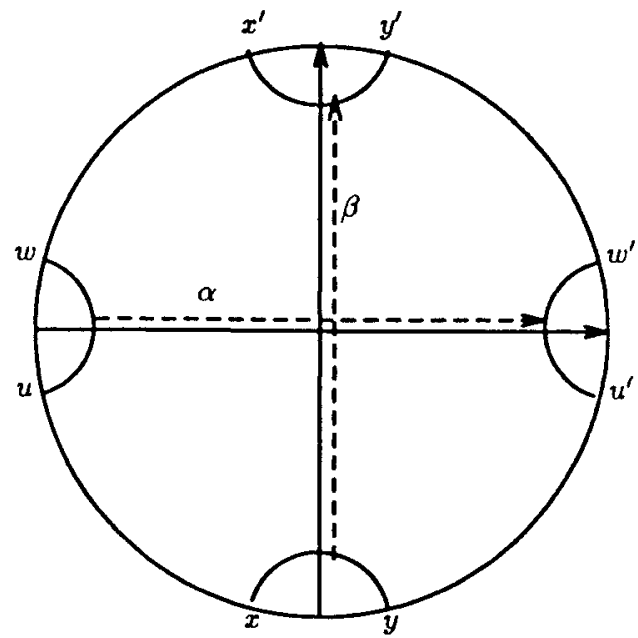

Fig. 1. A fundamental domain for the action of group $G$ is bounded by the isometric circles of $g$ and $h$ and their respective images under $g$ and $h$.

the error of our estimates. An implementation of that algorithm already exists [5]. The improvement given by formula (17) has not been included in that program yet.

In this section we consider an example of an implementation of this algorithm. To this end, let $g$ and $h$ be hyperbolic Möbius transformations mapping the unit disk onto itself in such a way that the axis of $g$ is the interval $[-1,1]$ and that of $h$ is $[-i, i]$. Suppose that the isometric circles of $g$ and $h$ do not intersect.

In our implementation of this algorithm we have considered the transformations

$$
\begin{aligned}
& g(z)=\frac{1001 z+999}{999 z+1001}, \\
& h(z)=\frac{1001 i z-999}{-999 z+1001 i} .
\end{aligned}
$$

Such transformations generate freely the Fuchsian group $G=\langle g, h\rangle$. The set $I$, that was used in our computations, consists, in this case, of the half-open arcs $[u, x),\left[y, u^{\prime}\right),\left[w^{\prime}, y^{\prime}\right)$, and $\left[x^{\prime}, w\right)$, taken in the positive direction of the unit circle. The mapping $g$ maps $u$ onto $u^{\prime}$ and $w$ onto $w^{\prime}, h$ maps $x$ onto $x^{\prime}$ and $y$ onto $y^{\prime}$. It follows that the image of $I$ on $\Omega(G) \cap \bar{D} / G$ is a simple closed curve.

It is actually easy to see that the quotient Riemann surface $D / G$ is a torus with one hole. Its double $\Omega(G) / G$ is a symmetric Riemann surface of genus 2. For any $r, 0<r<1$, the arcs $[-r, g(-r)]$ and $[-r i, h(-r i)]$ project onto simple closed geodesic curves $\alpha$ and $\beta$ on $\Omega(G) / G$ which form, together with their images under 
the symmetry of $\Omega(G)$, a canonical homology basis for $\Omega(G) / G$. Recall that $\Omega(G)$ is the domain of discontinuity of $G$. It is symmetric with respect to the unit circle: reflection in the unit circle maps $\Omega(G)$ onto itself. Figure 1 illustrates this group action.

Using Lemma 4 and its improvement given by formula (17) we then approximate the periods of differentials of this Riemann surface. Haataja has coded the present algorithm using both APL2 and the C-language. We do not go into the details of this code. We simply point out some of the practical problems and bottlenecks. They are:

- Precision of computation.

- The necessity of memory.

Observation 1. Precision of computation affects most importantly our possibilities of computing elements of group $G$. Let $g \in G$ be an element of the group whose word-length in terms of the generators and their inverses is $n_{g}$. Let $F_{G}$ be the fundamental domain of $G$ given in Fig. 1 .

Let $g_{1}, g_{2}, \ldots \in G$ be a sequence such that $n_{g_{j}} \rightarrow \infty$ as $j \rightarrow \infty$. Observe that the action of $G$ is such that

$$
m\left(g_{j}\left(F_{G}\right)\right) \rightarrow 0 \quad \text { as } j \rightarrow \infty .
$$

This implies that, as we compute the elements of $G$ with finite precision only, at some point all the elements $g_{j}$ are constant mappings, i.e., with finite precision of computation, we can consider only a finite part of $G$. As the precision grows, then that part also grows.

The importance of formula (17) lies in the fact that it allows us to compute effectively infinitely many terms of the sum (14). Therefore, by using (17) we may reduce the effect of Observation 1.

In our computation we have to replace $G$ by sets of the type

$$
G\langle m\rangle=\{g \in G \mid \text { word-length of } g \leq m\}
$$

In this simple case of a two generator free group,

$$
\# G\langle m\rangle=2\left(3^{m}-1\right)+1
$$

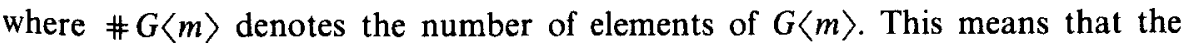
amount of disk space (in order to keep the whole set $G\langle m\rangle$ in memory at one time) grows exponentially with $m$. There is a recursive way around this problem, but that leads to an algorithm which is significantly slower. 
Let $\omega_{1}$ and $\omega_{2}$ be the base differentials, given by Lemma 3, corresponding to the generator $g$ and to $h$, respectively. Let

$$
A=\left(\begin{array}{ll}
\int_{\alpha} \omega_{1} & \int_{\alpha} \omega_{2} \\
\int_{\beta} \omega_{1} & \int_{\beta} \omega_{2}
\end{array}\right),
$$

where $\alpha$ and $\beta$ are the arcs indicated in Fig. 1. $A$ is not exactly the usual period matrix of the Riemann surface $\Omega(G) / G$, but a period matrix can be computed from $A$. We have computed approximations for $A$ only.

We have approximated the matrix $A$ using Lemma 4 and replacing $G$ with $G\langle m\rangle$ for $m=1, \ldots, 10$. In the list below, $A\langle m\rangle$ is the approximation obtained by replacing $G$ by $G\langle m\rangle$ :

$$
\begin{aligned}
& A\langle 1\rangle=\left(\begin{array}{cc}
-6.906846 & -0.230495-2.814504 i \\
-0.230495-2.814504 i & -6.906846
\end{array}\right), \\
& A\langle 2\rangle=\left(\begin{array}{cc}
-6.916535 & -0.000916-3.141053 i \\
-0.000916+3.141053 i & -6.916535
\end{array}\right), \\
& A\langle 3\rangle=\left(\begin{array}{cc}
-6.915768 & -0.000006-3.141592 i \\
-0.000006+3.141592 i & -6.915768
\end{array}\right), \\
& A\langle 4\rangle=\left(\begin{array}{cc}
-6.915763 & -3.141593 i \\
3.141593 i & -6.915763
\end{array}\right) .
\end{aligned}
$$

Next estimates $A\langle m\rangle, m=5,6, \ldots$, equal $A\langle 4\rangle$. We have not yet implemented the algorithms which estimate the errors of approximations.

Here is the output of the timing of the computation of $A\langle 4\rangle$ with a C-program written by Haataja running on an IBM RS6000 m 340 with 16 Mbytes of memory. These times include the computation of all the group elements up to the word-length 4 .

$$
\begin{array}{lr}
\text { \$ time periods } 4 \\
\text { real } & 0 \mathrm{~m} 0.47 \mathrm{~s} \\
\text { user } & 0 \mathrm{~m} 0.09 \mathrm{~s} \\
\text { sys } & 0 \mathrm{~m} 0.05 \mathrm{~s}
\end{array}
$$

\section{Acknowledgments}

This paper has benefited greatly from discussions with Kalevi Suominen, which the author gratefully acknowledges. 


\section{References}

1. N. L. Alling and N. Greenleaf. Foundations of the Theory of Klein Surfaces, Lecture Notes in Mathematics, vol. 219. Springer-Verlag, Berlin, 1971.

2. W. Burnside. On a class of automorphic functions. Proc. London Math. Soc., XXIII:49-88, 1891.

3. C. J. Earle and A. Marden. On Poincaré series with application to $h^{p}$ spaces of bordered Riemann surfaces. Illinois J. Math., 13:202-219, 1969.

4. B. H. Gross and J. Harris. Real algebraic curves. Ann. Sci. École Norm. Sup. (4), 14:157-182, 1981.

5. J. Haataja and M. Seppälä. Riemann, a program for computing periods of differentials, 1992. Preliminary version is available by anonymous ftp at sophie.helsinki.fi.

6. F. Klein. Über eine neue Art von Riemannschen Flächen. Math. Ann., 10, 1876.

7. H. Poincaré. Sur les Groupes des équations Linéaires. Acta Math., IV:201-312, 1884.

8. M. Seppälä. Moduli spaces of stable real algebraic curves. Ann. Sci. École Norm. Sup. (4), 24:519-544, 1991.

9. M. Seppälä and R. Silhol. Moduli spaces for real algebraic curves and real abelian varieties. Math. Z., 201:151-16S, 1989.

10. M. Seppälä and R. Silhol. Riemann surfaces, algebraic curves and their period matrices. Preprint, 1993.

11. Robert Silhol. Problèms de moduli en geométrie algébrique réelle. Manuscript, 1987.

12. R. Silhol. Compactifications of moduli spaces in real algebraic geometry. Invent. Math., 107:151$202,1992$.

Received October 12,1991, and in revised form January 8, 1992. 